\title{
Rotational dynamics of magnetic silica spheres studied by measuring the complex magnetic susceptibility
}

\author{
E M Claesson, B H Erné and A P Philipse \\ Van 't Hoff Laboratory for Physical and Colloid Chemistry, Utrecht University, Padualaan 8, \\ $3584 \mathrm{CH}$ Utrecht, The Netherlands \\ E-mail: e.m.claesson@chem.uu.nl
}

Received 25 February 2007

Published 19 June 2007

Online at stacks.iop.org/JPhysCM/19/286102

\begin{abstract}
The weak permanent magnetic dipole moment of cobalt ferrite-doped colloidal silica spheres was increased by exposure to a saturating magnetic field. The resulting change of the rotational dynamics of the magnetic microspheres in a weak alternating field was measured from low to high volume fraction in ethanol, using a frequency-dependent complex magnetic susceptibility setup. At low volume fractions, the increased dipolar attraction slows down Brownian rotation. At higher volume fractions, however, rotation is no longer slowed down as rapidly with increasing concentration, likely due to dipolar coupling between the particles which accelerates their partial alignment with the alternating field. This explanation is supported by the unexpected finding that salt addition accelerates particle rotation rather than slowing it down. At the highest volume fraction, colloidal crystals and glasses were formed in which only a small fraction of the spheres exhibit rotational mobility.
\end{abstract}

(Some figures in this article are in colour only in the electronic version)

\section{Introduction}

A widely applied experimental approach to determine the particle size in situ in colloidal dispersions is to measure the characteristic time or frequency of translational or rotational diffusion of the particles. This is usually done with optical techniques such as dynamic light scattering (DLS) [1], depolarized dynamic light scattering (DDLS) [2-4], time-resolved phosphorescence anisotropy (TPA) [5-7], and polarized fluorescence recovery after photobleaching (pFRAP) [8]. These techniques usually require particles which are nearly refractive index matched with their dispersion medium. Particles with a permanent electric [9] or magnetic dipole moment are usually highly light absorbing and cannot be optically matched in common solvents. With techniques like TPA and pFRAP, optical problems in more concentrated systems are avoided with so-called tracer particles, i.e. particles that are labelled 
with dye and which are dispersed in a more concentrated dispersion of non-labelled particles (hosts). In practice, synthesizing two different batches of particles that are chemically and physically identical, except for the dye, is difficult and, for the interpretation, a difference in size between tracer and host particles must be taken into consideration [10]. Here, we make use of a magnetic technique to study the concentration dependence of the rotational diffusion of interacting dipolar colloidal spheres dispersed in a liquid.

The expected trends for the qualitative effects of hydrodynamic and electrostatic interactions on the rotational diffusion of spheres have been well established in other publications $[2,3,11,12]$. In very dilute dispersions, the spheres do not influence each other, but when the concentration is increased, rotational diffusion is slowed down by hydrodynamic interaction: the rotation of one particle locally causes flow of the solvent around it, and this flow hinders the free rotation of neighbouring colloidal particles. The extent to which hydrodynamic interaction affects rotation depends on the total pair interaction potential of the spheres. Attraction locally causes spheres to approach each other, hence hydrodynamic interaction is increased and rotation slows down, whereas repulsion keeps the spheres apart and hydrodynamic interaction is counteracted. The quantitative relationship between the pair potential and rotational diffusion coefficient is not straightforward. Our aim is to gain new insight into that relationship by experimentally studying the effect of increasing attraction between dispersed colloidal spheres in an original and highly selective way.

Previously, systematic studies of the experimental factors affecting rotational diffusion always involved changes in the chemical composition of the colloidal dispersion. For example, the salt concentration was varied to affect the electrostatic repulsion [7]. In the present work, we study the rotational diffusion of recently developed colloidal silica spheres with embedded cobalt ferrite nanoparticles [13]. The spheres have a magnetic dipole moment in zero magnetic field and it can be permanently increased by exposure to a saturating magnetic field [14]. In this way, the attractive component of the total interaction potential is changed selectively, without any other change in the chemistry or physics of the system. This makes it possible to ascribe any resulting change in the rotation rate unambiguously to enhanced dipolar attraction. We present an experimental investigation of particle rotation in a colloidal system with repulsive as well as attractive interactions and qualitatively discuss the observed trends.

In section 2, a theoretical background is given to the rotational diffusion of spheres, interaction potentials and the complex magnetic susceptibility technique. After a description of experimental methods in section 3, a study of rotational dynamics is presented in section 4, based on complex magnetic susceptibility spectra. A general discussion of the results follows in section 5, leading to final conclusions in section 6.

\section{Theory}

\subsection{Rotational diffusion and interaction potentials}

For a free single spherical particle in a solvent with viscosity $\eta_{0}$, the rotational diffusion coefficient $D^{\mathrm{r}}$ is given by the Stokes-Einstein-Debye (SED) relation:

$$
D_{0}^{\mathrm{r}}=\frac{k_{\mathrm{B}} T}{8 \pi \eta_{0} R_{\mathrm{h}}^{3}},
$$

where $k_{\mathrm{B}} T$ is the thermal energy and $R_{\mathrm{h}}$ the hydrodynamic radius of the particle. At infinite dilution, the rotating colloids only experience the viscous drag by the solvent as quantified by (1). As the concentration of particles is increased, interactions with neighbouring particles come into play and the rotational diffusion coefficient is no longer described by the SED relation (1). For hard spheres, several theoretical studies of the concentration dependence of the 
rotational diffusion coefficient have been presented [3, 11, 15]. Cichocki et al [15] proposed an expression for the decrease in the rotational diffusion coefficient with increasing volume fraction $\phi$ :

$$
D^{\mathrm{r}}(\phi) / D_{0}=1-0.631 \phi-0.726 \phi^{2}
$$

For highly charged spheres, in deionized suspensions, Watzlawek and Nägele [12] proposed the following:

$$
D^{\mathrm{r}}(\phi) / D_{0}=1-1.28 \phi^{1.99}
$$

These expressions illustrate that Brownian rotation slows down as the concentration of particles increases and that this decay is slower for repulsive particles. The colloidal systems studied in this work consist of charged silica particles with weak magnetic dipolar interactions. In contrast to experiments with optical measuring techniques, the silica particles are dispersed in a non-matching solvent and van der Waals interactions should therefore be taken into account. The overall interaction potential for the particles used in our study may be represented as:

$$
U(r)=U_{\mathrm{HS}}(r)+U_{\mathrm{vdW}}(r)+U_{\mathrm{dip}}(r)+U_{\mathrm{el}}(r) .
$$

The first term is the hard-sphere potential, which prevents particle overlap:

$$
U_{\mathrm{HS}}(r)= \begin{cases}\infty & r<2 R \\ 0 & r>2 R\end{cases}
$$

$U_{\mathrm{vdW}}(r)$ is the attractive van der Waals interaction:

$$
U_{\mathrm{vdW}}(r)=-\frac{H}{12}\left[\frac{1}{(r / 2 R)^{2}-1}+\frac{1}{(r / 2 R)^{2}}+2 \ln \left(\frac{(r / 2 R)^{2}-1}{(r / 2 R)^{2}}\right)\right],
$$

where $H$ is the Hamaker constant, $R$ the particle radius and $r$ the centre-to-centre distance between two spheres.

The interaction between two permanent magnetic point dipoles in arbitrary orientations is given by:

$$
U_{\text {dip }}(r)=\frac{\mu_{0} \mu_{1} \mu_{2}}{4 \pi r^{3}}\left[\hat{\mu}_{1} \cdot \hat{\mu}_{2}-3\left(\hat{\mu}_{1} \cdot \hat{r}\right)\left(\hat{\mu}_{2} \cdot \hat{r}\right)\right]
$$

where $\mu_{0}=4 \pi \times 10^{-7} \mathrm{~J} \mathrm{~A}^{-2} \mathrm{~m}^{-1}, \mu_{1}$ and $\mu_{2}$ are the magnetic dipole moments of spheres 1 and $2, \hat{\mu}_{1}$ and $\hat{\mu}_{2}$ are unit vectors pointing along the directions of the dipoles of particles 1 and 2 , and $\hat{r}$ is a unit vector that points in the direction from the centre of particle 1 to that of particle 2. When the prefactor $\mu_{0} \mu_{1} \mu_{2} /\left(4 \pi r^{3}\right)$ is small compared to $k_{\mathrm{B}} T$ and $\mu_{1}=\mu_{2}=\mu$, the Boltzmann-weighted average of $U_{\text {dip }}$ is given by:

$$
\left\langle U_{\text {dip }}(r)\right\rangle=-\frac{2\left(\mu_{0} \mu^{2}\right)^{2}}{3 k_{\mathrm{B}} T(4 \pi)^{2} r^{6}} .
$$

The Boltzmann weights $\exp \left[-U_{\mathrm{dip}} /\left(k_{\mathrm{B}} T\right)\right]$ depend on the orientation according to (7). This is the classical formula for the pair potential of permanent dipoles [16]. In (8) it is assumed that only a weak statistical preference exists for the head-to-tail dipole-dipole configuration and that the interaction can be treated as an isotropic attraction.

The electrostatic term for the interaction between colloidal spheres with a constant surface charge $Z e$ is:

$$
U_{\mathrm{el}}(r)=\frac{(Z e)^{2}}{\epsilon_{0} \epsilon_{\mathrm{r}}}\left[\frac{\exp (\kappa R)}{1+\kappa R}\right]^{2} \frac{\exp (-\kappa R)}{r}
$$


in which the inverse Debye screening length $\kappa$ for a monovalent salt is given by:

$$
\kappa=\sqrt{\frac{2 e^{2} c_{\mathrm{s}}}{\epsilon_{0} \epsilon_{\mathrm{r}} k_{\mathrm{B}} T}},
$$

where $e$ is the elementary charge, $\epsilon_{0}$ is the vacuum permittivity, $\epsilon_{\mathrm{r}}$ is the dielectric constant of the solvent (for ethanol, $\epsilon_{\mathrm{r}}=25.3$ [17]) and $c_{\mathrm{s}}$ is the salt concentration given in number of cations or anions per volume.

\subsection{Complex magnetic susceptibility}

To monitor the rotational diffusion in a colloidal system of isotropic particles, these must be anisotropically labelled. For DDLS, the particles should have an optically anisotropic crystalline core and the relaxation of the labels is measured by monitoring the evolution of the depolarized component of the scattered intensity [2]. With techniques such as TPA or pFRAP, an anisotropic label is created by illuminating fluorescent particles with a polarized light beam. The decay of the polarized phosphorescence or the fluorescence emission is ascribed to orientational relaxation of the particles and is measured in time $[5,8]$.

For complex magnetic susceptibility spectroscopy, an anisotropic probe is present in the form of a permanent magnetic dipole moment inside the colloids. With the highly sensitive setup used here, particles with a low magnetic content can be studied at low concentrations. The magnetic nanoparticles embedded inside the microparticles act as a probe to measure the diffusive reorientation.

The complex magnetic susceptibility as a function of the radial frequency, $\omega$, is defined as:

$$
\chi(\omega)=\frac{M(\omega)}{H(\omega)},
$$

where $H(\omega)$ is the applied alternating magnetic field and $M(\omega)$ is the resulting harmonic magnetization. For colloidal particles with a permanent magnetic dipole moment, frequencydependent measurements of $\chi$ at low $H(\omega)$ field amplitude yield the rotational diffusion rate of the particles [14]. The characteristic frequency $\omega_{\mathrm{B}}$ is obtained from spectra of the real and imaginary components of the complex susceptibility:

$$
\begin{aligned}
& \chi^{\prime}(\omega)=\chi_{\mathrm{HF}}+\chi_{\mathrm{LF}} \frac{\omega_{\mathrm{B}}^{2}}{\omega_{\mathrm{B}}^{2}+\omega^{2}} \\
& \chi^{\prime \prime}(\omega)=\chi_{\mathrm{LF}} \frac{\omega_{\mathrm{B}} \omega}{\omega_{\mathrm{B}}^{2}+\omega^{2}} .
\end{aligned}
$$

Here, $\chi_{\mathrm{HF}}$ is the high-frequency limit and $\chi_{\mathrm{LF}}+\chi_{\mathrm{HF}}$ is the low-frequency limit of the susceptibility. Whereas $\chi_{\mathrm{HF}}$ is due to nanoparticles whose magnetic moment is not blocked inside the nanocrystals, $\chi_{\mathrm{LF}}$ scales with the volume fraction, $\phi$, of magnetic silica particles and the square of their dipole moment $\mu_{\mathrm{mp}}$ :

$$
\chi_{\mathrm{LF}}=\frac{N_{\mathrm{mp}}}{V} \frac{\mu_{0}\left(\mu_{\mathrm{mp}}\right)^{2}}{3 k_{\mathrm{B}} T}=\frac{\phi}{V_{\mathrm{p}} V} \frac{\mu_{0}\left(\mu_{\mathrm{mp}}\right)^{2}}{3 k_{\mathrm{B}} T},
$$

where $\omega=2 \pi f$ is the angular frequency in $\mathrm{s}^{-1}, f$ is the cycle frequency in $\mathrm{Hz}, N_{\mathrm{mp}}$ is the number of microparticles per sample volume, $V$ is the volume of the entire sample and $V_{\mathrm{p}}$ is the volume of a single microparticle. With this technique, the magnetic contribution of the microparticles, $\chi_{\mathrm{HF}}$, is separated from other contributions to the susceptibility such as the field-induced alignment of nanoparticle dipoles that are not blocked or of free nanoparticles, which are not embedded in microparticles. Néel as well as Brownian relaxation of single 
nanoparticles occur at much higher frequencies than the frequency of Brownian rotation of the microspheres [14].

The characteristic frequency, $\omega_{\mathrm{B}}$, is directly related to the rotational diffusion coefficient of the particles [18]:

$$
\omega_{\mathrm{B}}=2 D^{\mathrm{r}} .
$$

Polydispersity is taken into account by fitting the results to a lognormal distribution of the characteristic frequency:

$$
P\left(\omega_{\mathrm{B}}\right)=\frac{1}{\sigma \omega_{\mathrm{B}} \sqrt{2 \pi}} \exp \left[\frac{-\ln ^{2}\left(\omega_{\mathrm{B}} / \omega_{0}\right)}{2 \sigma^{2}}\right] .
$$

Here, $\sigma^{2}$ is the variance and $\omega_{0}$ is the value of $\omega_{\mathrm{B}}$ at the maximum of the distribution. Measurement of the susceptibility in the low-frequency limit on a sample with a known concentration $N_{\mathrm{mp}} \mathrm{V}^{-1}$ allows calculation of $\mu_{\mathrm{mp}}$, which means that the average dipoledipole interaction potential in the system can be estimated, based on the obtained value of $\mu_{\mathrm{mp}}$ in (14) [14].

\section{Experimental details}

Two samples of silica colloids were studied (see table 1), both with embedded cobalt ferrite magnetic nanoparticles: in one sample the particles have a thin $\sim 10 \mathrm{~nm}$ silica layer around the magnetic particles and in the other this layer is $\sim 30 \mathrm{~nm}$. It is expected that a thicker silica layer will reduce the magnetic interaction between spheres at particle contact. In the limiting case that the magnetic contact attraction is much weaker than $k_{\mathrm{B}} T$, the magnetic core will only serve as a probe for measuring the Brownian rotation of the microparticles, whereas for particles with a thin layer of silica, the dipole-dipole interactions can still influence the rotational diffusion of the particles.

\subsection{Sample preparation}

The chemical synthesis of silica colloids with embedded magnetic nanoparticles has been described in detail in [13]. In brief, cobalt ferrite nanoparticles were prepared in a coprecipitation step [19] and attached to Stöber silica particles [20] provided with thiol surface groups. The resulting silica-ferrite composites were coated with an outer silica shell, and the particle surface was grafted covalently with 3-(trimethoxysilyl)propyl methacrylate (TPM). The particle dispersions were purified by repeated sedimentation under gravity and redispersion in ethanol. The particle radius, $R_{\mathrm{TEM}}$, and the silica shell thickness, $\Delta R_{\mathrm{Si}}$, were obtained from image analysis of electron microscopy pictures (see figure 1). The magnetic dipole moment of the silica-cobalt ferrite particles can be increased permanently by magnetic treatment (see table 2) in a strong magnetic field [14]. After susceptibility measurements on the as-synthesized magnetic silica particles, they were magnetized in a homogeneous field of $2 \mathrm{~T}$ using a Bruker BE $25 \mathrm{v}$ electromagnet, after which the susceptibility measurements were repeated. The magnetized sample was also measured after re-dispersion in ethanol with $10 \mathrm{mM} \mathrm{LiNO}_{3}$.

A small amount of each dispersion with known weight $m_{\text {disp }}$ and volume $V_{\text {disp }}$ was dried to obtain the weight of dry particles, $m_{\mathrm{p}}$. $V_{\text {disp }}$ was obtained by measuring the height of the dispersion with a cathetometer inside a calibrated tube with a known volume-height relation. Together with the solvent density, $\rho_{\mathrm{s}}=0.789 \mathrm{~g} \mathrm{ml}^{-1}$ [17], the mass density of the particles, 

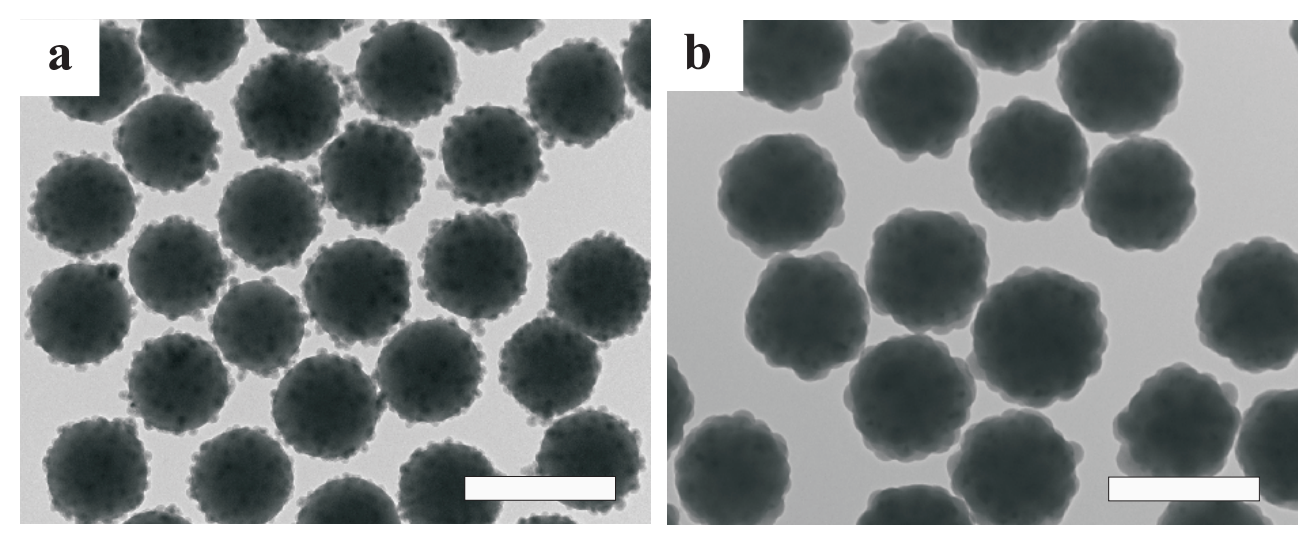

Figure 1. Transmission electron microscopy pictures of the studied particles: (a) silica-cobalt ferrite microspheres (sample A) with a silica shell thickness of $\sim 10 \mathrm{~nm}$ and (b) silica-cobalt ferrite microspheres (sample B) with a silica shell thickness of $\sim 30 \mathrm{~nm}$. The scale bars correspond to $500 \mathrm{~nm}$.

Table 1. Characteristics of the magnetic silica dispersions.

\begin{tabular}{lcc}
\hline Sample code & Sample A & Sample B \\
\hline Physical radius, $R_{\mathrm{TEM}}(\mathrm{nm})$ & $187 \pm 34$ & $207 \pm 12$ \\
Hydrodynamic radius, $R_{\mathrm{h}}(\mathrm{nm})^{\mathrm{a}}$ & $230 \pm 30$ & $286 \pm 47$ \\
Silica shell thickness, $\Delta R_{\mathrm{Si}}(\mathrm{nm})$ & $\sim 10$ & $\sim 30$ \\
Particle mass density, $\rho_{\mathrm{p}}\left(\mathrm{g} \mathrm{ml}^{-1}\right)$ & 2.5 & 1.8 \\
\hline
\end{tabular}

${ }^{a}$ Calculated from (15) and (1) at the lowest measured concentration.

Table 2. Properties of the magnetic silica dispersions before and after magnetization at $2 \mathrm{~T}$.

\begin{tabular}{llllll}
\hline & \multicolumn{2}{c}{ Sample $\mathrm{A}$} & & \multicolumn{2}{c}{ Sample B } \\
\cline { 2 - 3 } & Before mag. & After mag. & & Before mag. & After mag. \\
\hline Dipole moment, $\mu\left(\mathrm{A} \mathrm{m}^{2}\right)$ & $2.5 \times 10^{-17}$ & $3.6 \times 10^{-17}$ & & $2.7 \times 10^{-17}$ & $3.1 \times 10^{-17}$ \\
Max. dipole-dipole int. (7), $U_{\max }\left(k_{\mathrm{B}} T\right)$ & 0.59 & 1.2 & & 0.49 & 0.65 \\
Interaction with 57 A m
\end{tabular}

$\rho_{\mathrm{p}}$, was calculated from:

$$
\rho_{\mathrm{p}}=\frac{m_{\mathrm{p}}}{V_{\mathrm{disp}}-\left(\frac{m_{\mathrm{disp}}-m_{p}}{\rho_{\mathrm{s}}}\right)} .
$$

The sample volume, $V$, was obtained in the same way as described above and the volume fraction, $\phi$, of particles in stock dispersions of all samples was calculated:

$$
\phi=\frac{\phi_{\mathrm{m}} m_{\mathrm{disp}}}{V \cdot \rho_{p}},
$$

where $\phi_{\mathrm{m}}$ is the mass fraction of a stock dispersion. The measured value of $\chi_{\mathrm{HF}}$ is directly proportional to the number of Neél-relaxing particles in the sample. Since the ratio between the number of microparticles and the number of Neél particles is constant for particles from the same synthesis batch, $\chi_{\mathrm{HF}}$ can be used as an in situ measure for the volume fraction (see figure 6). The samples were diluted by stepwise addition of small aliquots of ethanol, followed by thorough homogenization. Conductivity measurements in the supernatant after 
sedimentation as well as in a semi-dilute dispersion of both samples $\mathrm{A}$ and $\mathrm{B}$ showed a salt concentration of approximately $0.2 \mathrm{mM}$, which corresponds to a Debye length $\kappa^{-1} \approx$ $12 \mathrm{~nm}$ (10). Salt was added to the magnetized sample A by repeated centrifugation of the particles and re-dispersion in ethanol with $10 \mathrm{mM} \mathrm{LiNO}_{3}$. This salt concentration decreases the Debye length to $1.7 \mathrm{~nm}$.

\subsection{Complex magnetic susceptibility measurements}

At each concentration, a frequency-dependent complex magnetic susceptibility spectrum was measured. For the fully sedimented samples, the spectra were measured starting from $500 \mathrm{~Hz}$ down to $0.1 \mathrm{~Hz}$. However, for the diluted samples, for which the characteristic frequency is found at higher frequencies, the spectra were measured from $500 \mathrm{~Hz}$ down to $1 \mathrm{~Hz}$. This was done to shorten the measurement time, thereby limiting the effect of sedimentation on the spectrum. Control spectra were also measured the other way around, from 1 to $500 \mathrm{~Hz}$, to verify that the characteristic frequency remained the same, which was the case.

Complex magnetic susceptibility measurements were performed using a setup consisting of a differential transformer with two concentric multilayered cylindrical copper coils, with primary layers through which an applied alternating current flows and secondary layers in which an alternating voltage is induced. Secondary coils were used with a maximized number of layers beyond which the signal would no longer increase but decrease due to capacitance and absorption effects. An ac current is applied to the primary coils using a Yokogawa FG120 function generator, and the voltage induced in the secondary coils is measured using a 7265 Perkin-Elmer differential lock-in amplifier in the $0.1 \mathrm{~Hz}$ to $1 \mathrm{kHz}$ range. When a sample is introduced into the coils, the change in the measured alternating voltage is proportional to the complex susceptibility of the sample. The amplitude of the alternating magnetic field is very low. The measurements here were performed at $57 \mathrm{~A} \mathrm{~m}^{-1}$ if not specified otherwise. A more detailed description of the setup will be presented elsewhere [21]. The coils with the sample were kept inside an isolated box with a constant temperature, $T=295.5 \mathrm{~K}$.

For the highest volume fractions, the aspect ratio of the sample in the tube was of order 1 , resulting in a demagnetization of $\sim 10 \%$. The measured absolute values of $\chi$ were corrected for demagnetization effects using [22]:

$$
\chi_{\text {real }}=\frac{\chi_{\text {meas }}}{1-N_{\mathrm{f}} \chi_{\text {meas }}}
$$

where $N_{\mathrm{f}}$ is a correction factor obtained from [22] which depends on the aspect ratio of the cylindrical sample. This correction is only approximate, since the cylindrical sample tube had a rounded bottom. The measured value of the characteristic frequency is not influenced by demagnetization effects.

\section{Results}

A selection of the complex magnetic susceptibility spectra measured on dispersions of magnetic silica particles is shown in figures 2 and 3. The solid lines in these figures are least-squares fits of the spectra based on equations (12), (13) and a lognormal distribution of the characteristic frequency $\omega_{\mathrm{B}}$ around a value of $\omega_{0}$ with a variance $\sigma^{2}(16)$ [23].

In figure 2, susceptibility spectra are shown for samples A and B at the same concentration before and after magnetization of the sample. For the particles with a thin silica shell (A), the increase in dipole moment results in a slight shift of the characteristic frequency to lower frequencies, $\omega_{0}$, and hence to a lower rotational diffusion coefficient $D_{\mathrm{r}}$. For the particles with 


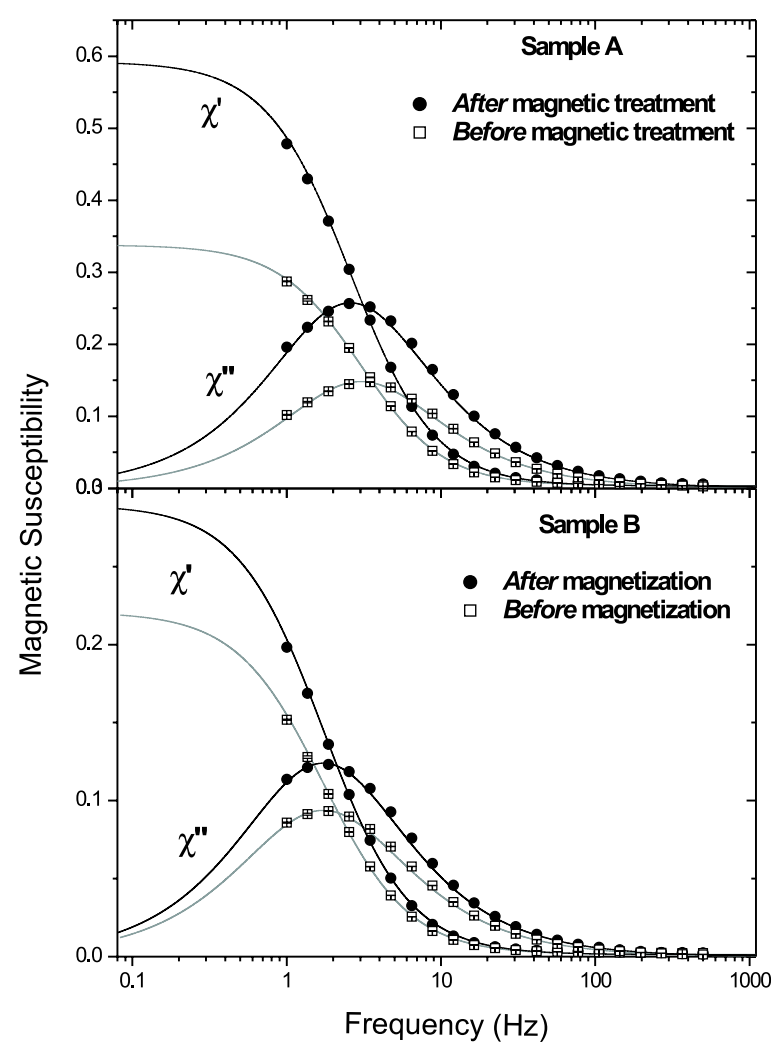

Figure 2. Frequency-dependent complex magnetic susceptibility spectra at moderate concentrations for samples A $(\phi=0.12)$ and $\mathrm{B}(\phi=0.13)$ before and after magnetization of the sample at $2 \mathrm{~T}$. The solid lines are least-squares fits of the spectra. The increase in $\chi_{\mathrm{LF}}$ shows that the permanent dipole moment of the microparticles has been increased.

a thicker silica shell (B), this effect is not observed, as a consequence of the thicker silica shell in combination with a smaller increase in dipole moment.

In figure 3 an example is shown of how the susceptibility spectrum changes upon dilution of a sample. At low concentrations, the spectra show a narrow distribution of $\omega_{\mathrm{B}}$ and thus a narrow distribution of $D_{\mathrm{r}}$ (see equation (15)). For the fully sedimented and partially crystalline samples, this distribution is broadened and the mean characteristic frequency and rotational diffusion coefficient are significantly lower. The broadening of the spectra at higher volume fractions is probably due to a polydispersity effect that becomes more important as the particles come closer together. In an ordered structure of particles, a small particle will be more free to rotate than its larger neighbours. This gives, averaged over a whole sample volume, a larger distribution of $\omega_{0}$.

The rotational diffusion coefficients obtained from the susceptibility spectra are displayed in figure 4 as a function of the volume fraction. The corresponding widths of the distributions of the characteristic frequency can be found in figure 5. In the most dilute regime of sample A, the characteristic frequency is $\omega_{0} /(2 \pi) \approx 3.7 \mathrm{~Hz}$ before and after magnetization. This value decreases rapidly up to $\phi \sim 0.01$ for the magnetized sample.

At concentrations $\phi>0.01$, the decrease in $\omega_{0}$ as a function of $\phi$ is slower and parallel for sample A before and after magnetization. Finally, at the highest concentrations, where the 


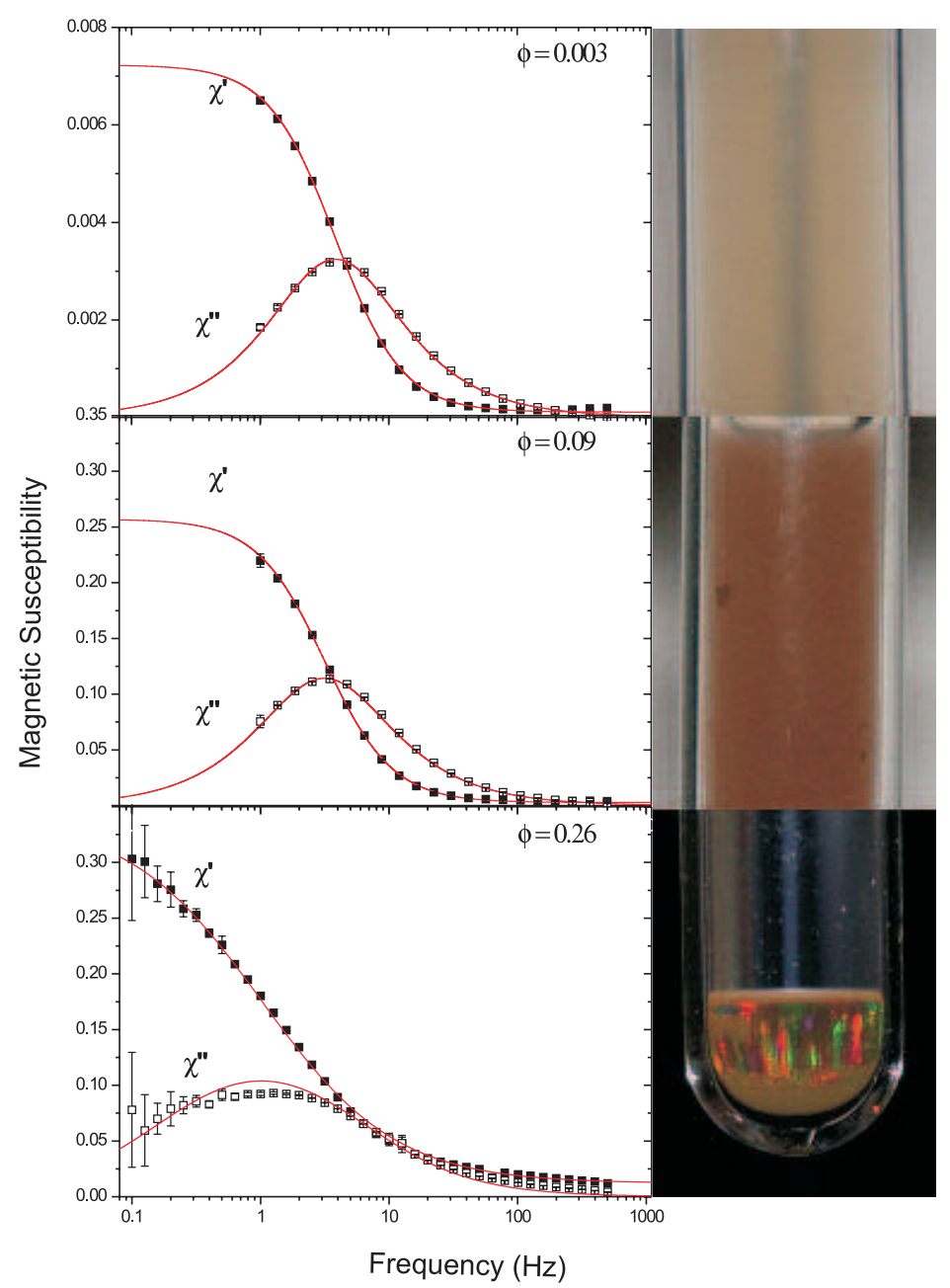

Figure 3. Frequency-dependent complex magnetic susceptibility spectra and photographs of the dispersion of sample A from high dilution $(\phi=0.003)$ to partially crystalline sediment $(\phi=0.26)$. For the sediment, the characteristic frequency is shifted to a lower value and the distribution of $\omega_{\mathrm{B}}$ is broadened compared to the diluted samples.

particles are fully sedimented and form partially a colloidal crystal and partially a colloidal glass, there is again a drop in $\omega_{0}(\phi)$, accompanied by a steep increase in the distribution width of $\omega_{\mathrm{B}}$ as the particles become restricted in their motion. A similar result is obtained when a colloidal glass phase is created by centrifugation of a dispersion at $20 \mathrm{~g}$. Since the only difference between these two measurement series is the increase in the microparticle dipole moment, obtained by treatment in an external magnetic field, the difference in the two $\omega_{0}(\phi)$ profiles must be explained in terms of increasing dipolar attractions, as will be discussed in section 5. Figure 4 also shows the data obtained for the magnetized sample A after the addition of $10 \mathrm{mM} \mathrm{LiNO}_{3}$, which at low concentration leads to faster rotation than without added salt.

Figure 6 shows how the low-frequency limit of the susceptibility spectrum, $\chi_{\mathrm{LF}}$, increases linearly with the susceptibility in the high-frequency limit, $\chi_{\mathrm{HF}}$. $\chi_{\mathrm{LF}}$ is proportional to the number concentration of single microparticles (14) and $\chi_{\mathrm{HF}}$ is proportional to the number 

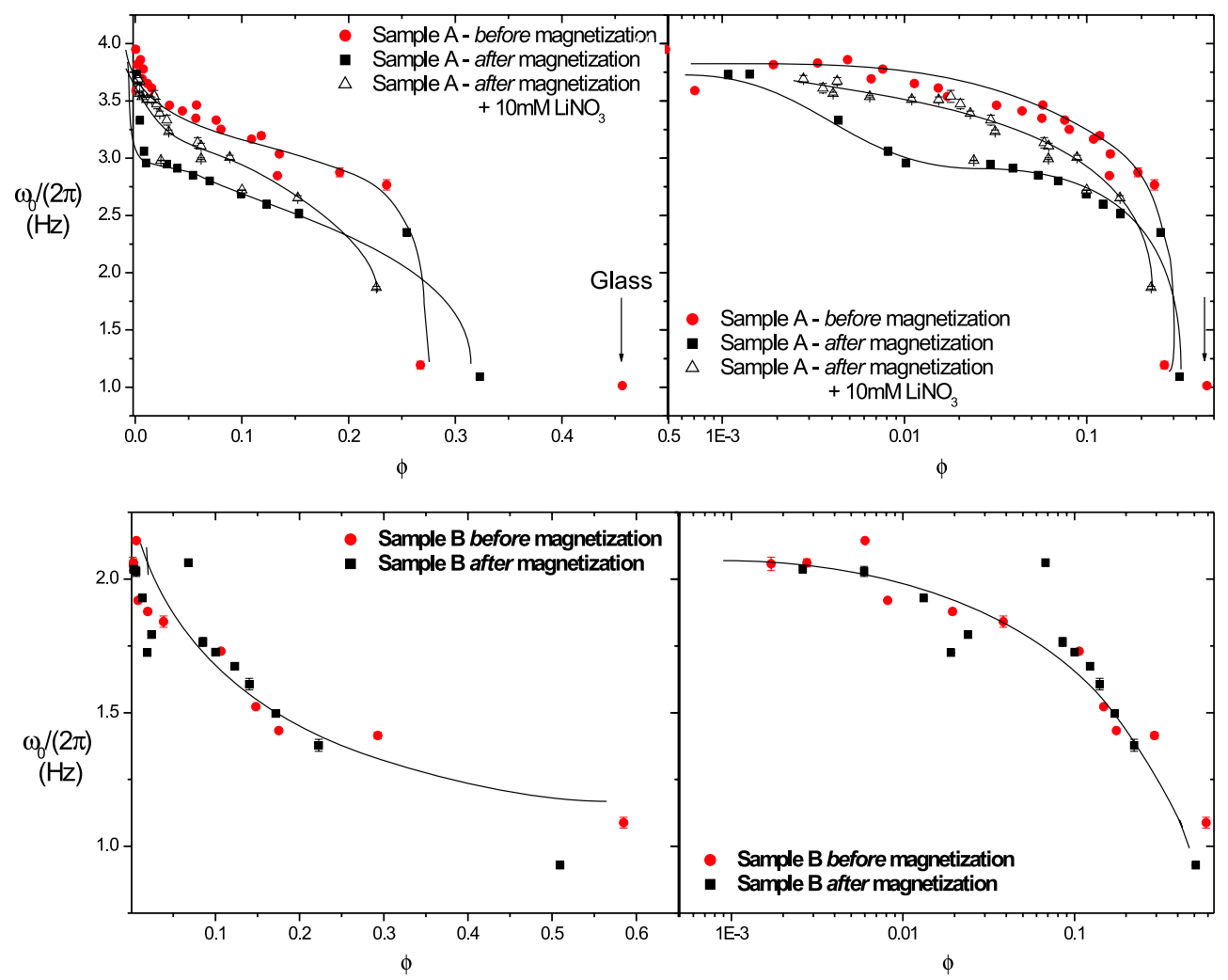

Figure 4. The characteristic frequency, $\omega_{0} /(2 \pi)$, as a function of volume fraction for samples $\mathrm{A}$ and B: left, linear scale; right, logarithmic scale. The arrow indicates a point measured on a colloidal glass of the particles in sample A before magnetization, obtained by sedimentation in a centrifuge at $20 \mathrm{~g}$. The solid curves are a guide to the eye.

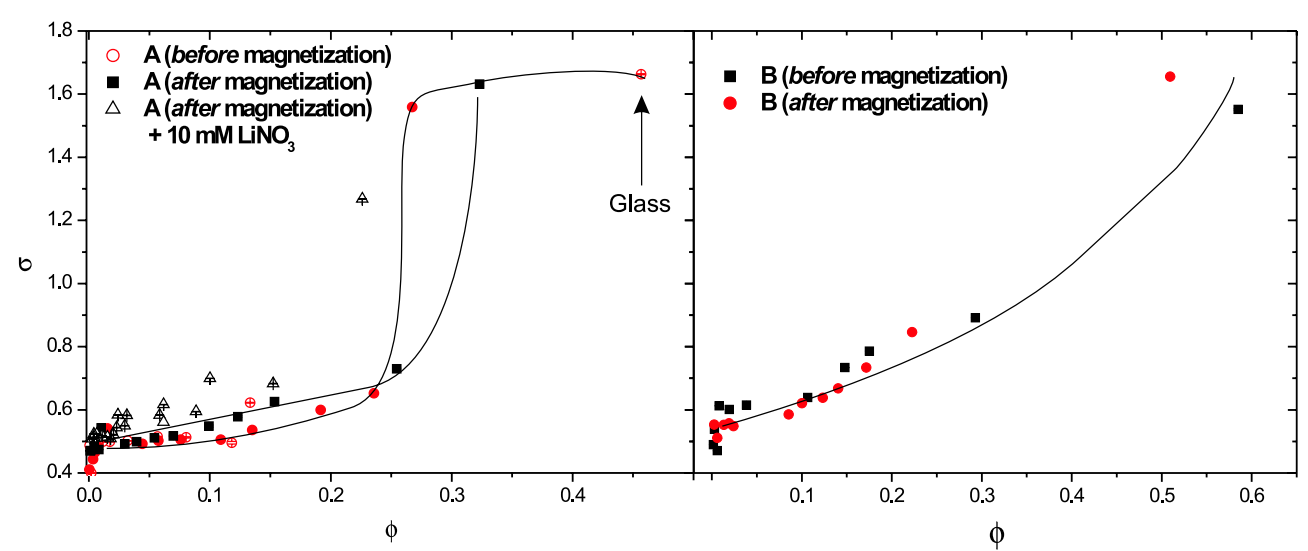

Figure 5. The distribution width, $\sigma$, of the characteristic frequency as a function of volume fraction (16). The solid curves are a guide to the eye.

of Néel-relaxing particles. The deviation from linearity at the two highest concentrations indicates that a fraction of the microspheres is no longer as free to rotate and therefore no 


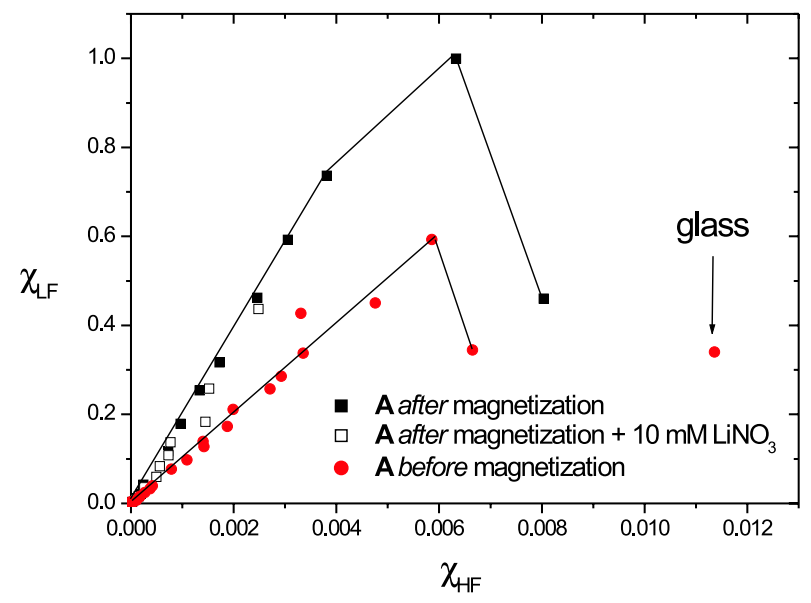

Figure 6. The low-frequency limit of the susceptibility spectrum, $\chi_{\mathrm{LF}}$, as a function of the susceptibility in the high-frequency limit, $\chi_{\mathrm{HF}}$, which is proportional to $\phi$. The deviation from linearity at the two highest concentrations indicates that a fraction of the microspheres is no longer free to rotate and therefore does not contribute to $\chi_{\mathrm{LF}}$. The solid lines are a guide to the eye, and the arrow indicates the point measured on the particles from sample A in a glassy state obtained by centrifugation at $20 \mathrm{~g}$.

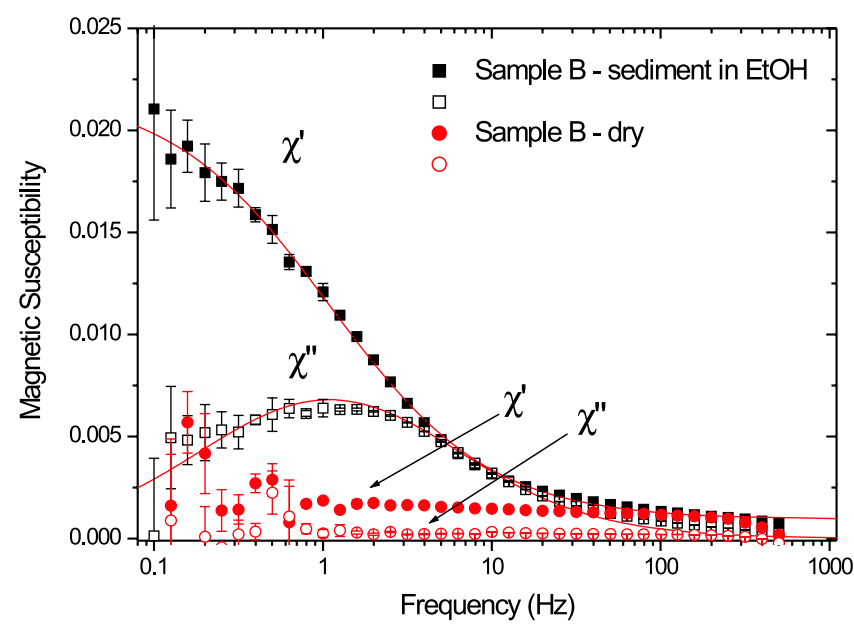

Figure 7. Frequency-dependent complex magnetic susceptibility spectra for the sedimented particles in sample B and the same sample as a dry powder after evaporation of the solvent. In the sediment of sample B, a fraction of the particles is restricted from moving, as was shown in figure 6. However, it is clear that the sediment still has a much higher susceptibility than when the particles are not moving at all, as in the dry powder. The microparticles in the powder no longer exhibit Brownian motion and, as a result, only the Néel-relaxing nanoparticles are detected, with a much weaker frequency dependence, since the characteristic frequency of the Néel relaxation is much higher.

longer contributes to $\chi_{\mathrm{LF}}$. In figure 7 , a susceptibility spectrum is shown before and after a full removal of solvent by evaporation. The particles in the dry powder are not moving and, as a result, only the $\chi_{\mathrm{HF}^{-}}$-component of the complex magnetic susceptibility remains. This confirms that the characteristic frequencies displayed in the previous figures correspond to rotation of the colloidal particles. 


\section{Discussion}

\subsection{Magnetic properties and magnetic interactions}

The rotational diffusion of the magnetic silica spheres is clearly affected by their interaction potential: exposure of the particles to a saturating magnetic field increases their magnetic moment and decreases the characteristic frequency, $\omega_{0}$ (see figure 4). Two questions arise concerning the magnetic dipole interaction between the type of composite particles used in our experiments: (a) is the interaction sufficiently weak that it can be treated as an effectively isotropic interaction and (b) what is the influence of the fact that the particles are not point dipoles but instead have a shell of magnetic nanoparticles?

5.1.1. Attractive magnetic dipole interactions. The interaction between two permanent magnetic point dipoles in arbitrary orientations is given by (7). When the prefactor $\mu_{0} \mu^{2} /\left(4 \pi r^{3}\right)$ exceeds $k_{\mathrm{B}} T$, the head-to-tail configuration starts to dominate, especially at high concentrations, and experiment and theory indicate the formation of dipolar chains consisting of two or more particles [24-26].

The maximum dipolar interaction energy is achieved when two particles are in contact and when the particle dipole moments are aligned in the head-to-tail configuration: $U_{\max }=$ $\mu_{0} \mu^{2} /\left(2 \pi d^{3}\right)(7)$. For our particles, $U_{\max }$ lies clearly below $2 k_{\mathrm{B}} T$, even after treatment in a saturating magnetic field (table 2), indicating that long-lived dipolar structures are absent. Furthermore, from the characteristic frequencies of the order of $4 \mathrm{~Hz}$, the formation of dipolar structures that exist longer than about $40 \mathrm{~ms}$ can be excluded, based on the experimental susceptibility spectra. The smallest dipolar structures would be doublets, whose rotational diffusion can be approximated by that of a sphero-cylinder of aspect ratio $L / d=2$, leading to a characteristic frequency lower than that of a single sphere by a factor of five [27]. In figure 8, calculated complex magnetic susceptibility spectra are shown for a simple shift of the characteristic frequency without doublet formation (figure 8(A)) and for the appearance of doublets at the cost of single particles (figure $8(\mathrm{~B})$ ). These can be compared to the experimentally obtained spectra, of which one example is given in figure $8(\mathrm{C})$. In the experiments, the characteristic frequency remains around the value expected for single sphere rotation (equations (1) and (15)) and the shifts in $\omega_{\mathrm{B}}$ are simple shifts similar to what is shown in figure 8(A). We conclude that long-lived dipolar structures, i.e. structures that would rotate as new entities rather than as separate spheres, are absent in our colloidal dispersions. It therefore seems reasonable to assume that the magnetic interaction can be treated as an isotropic attraction whose distance dependence is given by (8).

5.1.2. Effect of position of the magnetic nanoparticles inside the microspheres. The second issue affecting magnetic dipole attraction between the silica spheres is that they are not point dipoles but have a shell of magnetic nanoparticles. The magnetic dipole moment of a silica particle is given by the vector sum of the dipoles in the magnetic shell [14]. In the case of two silica dipolar particles with all nanoparticle dipoles parallel to each other in the head-to-tail configuration, it can be seen qualitatively why the attraction is stronger when the nanoparticles are in a shell (figure 9(A)) rather than at the centre of the particle (figure 9(B)). Compared to the case where the nanodipoles are at the centre of the microparticles, the weak attractions between far-removed dipoles are more than compensated by the strong interactions between nearby nanoparticle dipoles, due to the $r^{-3}$-dependence of the interaction between dipoles (7).

Numerical calculations were performed of the dipolar interaction between composite spheres, as in figure 9(A), with an outer diameter $d_{1}=375 \mathrm{~nm}$, a microparticle dipole moment 


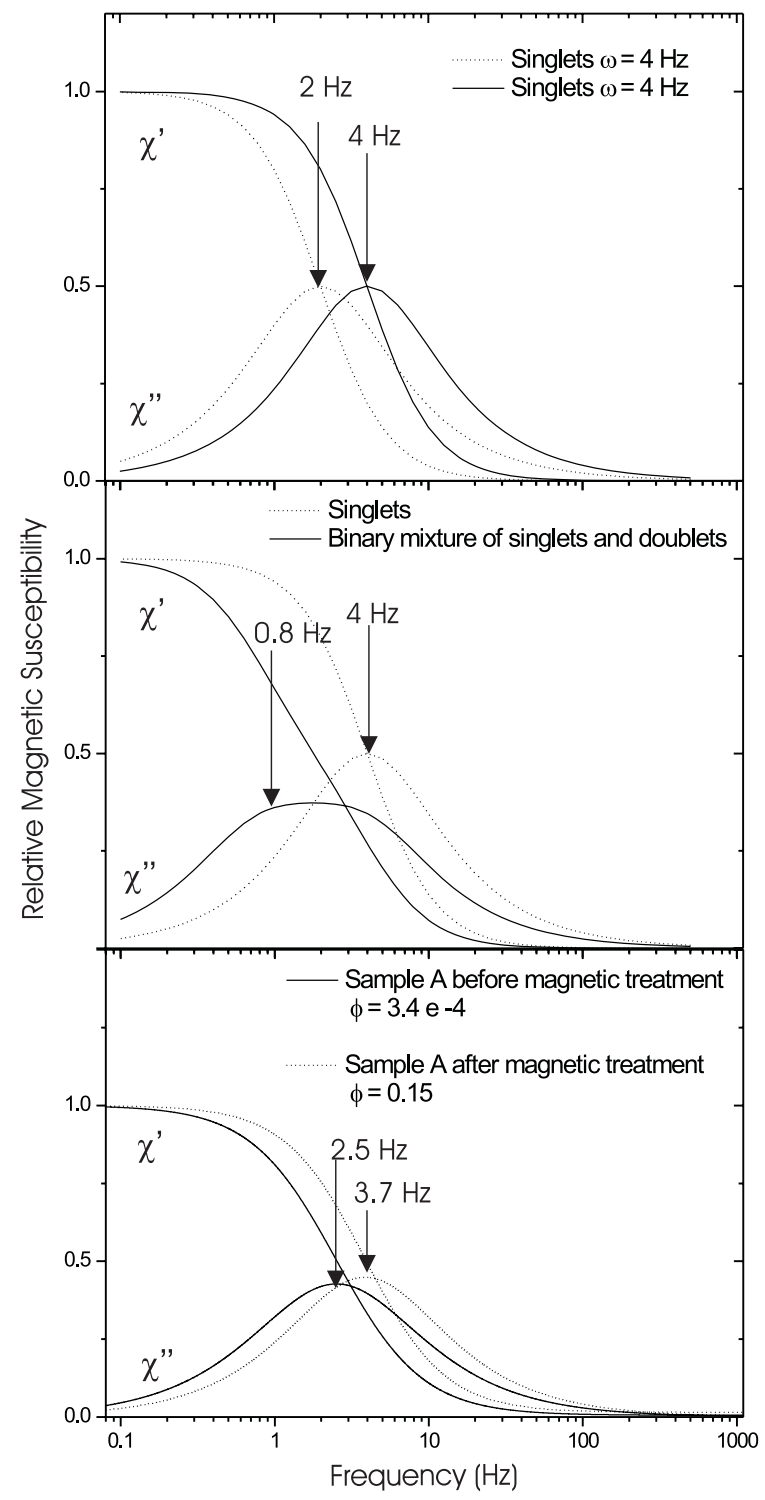

Figure 8. The effect of (A) a simple shift of the characteristic frequency of a susceptibility spectrum of single particles of $R_{\mathrm{h}}=230 \mathrm{~nm}$ without formation of doublets, (B) the effect on the susceptibility spectrum upon formation of doublets at the expense of singlets and (C) experimentally obtained spectra for dilute sample A before magnetic treatment and at $\phi=0.15$ for the same particles after magnetic treatment. In the experimental curves, the shift to lower frequencies at the higher concentration is not large enough to correspond to doublets or larger structures.

of $4 \times 10^{-17} \mathrm{~A} \mathrm{~m}^{2}$ and 342 parallel magnetic nanoparticle dipoles evenly distributed on an inner shell of diameter $d_{2}=355 \mathrm{~nm}$. This roughly corresponds to the particle in sample A, where the outer silica shell is only $\sim 10 \mathrm{~nm}$. The weighted average interaction $\left\langle U_{\text {dip }}\right\rangle$ was calculated with Boltzmann weights $\exp \left[-U_{\mathrm{dip}} /\left(k_{\mathrm{B}} T\right)\right]$ by adding the contributions of all pair interactions between nanoparticle dipoles, taking into account their orientations and spatial positions. At each distance between the microparticles, the weighted average interaction was 
A.

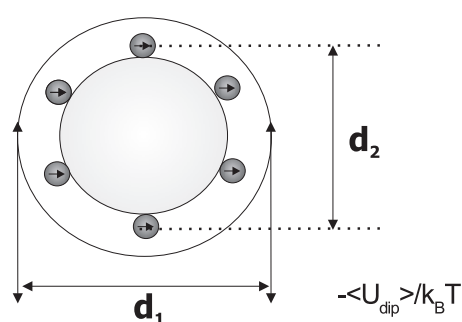

Particle contact

B.
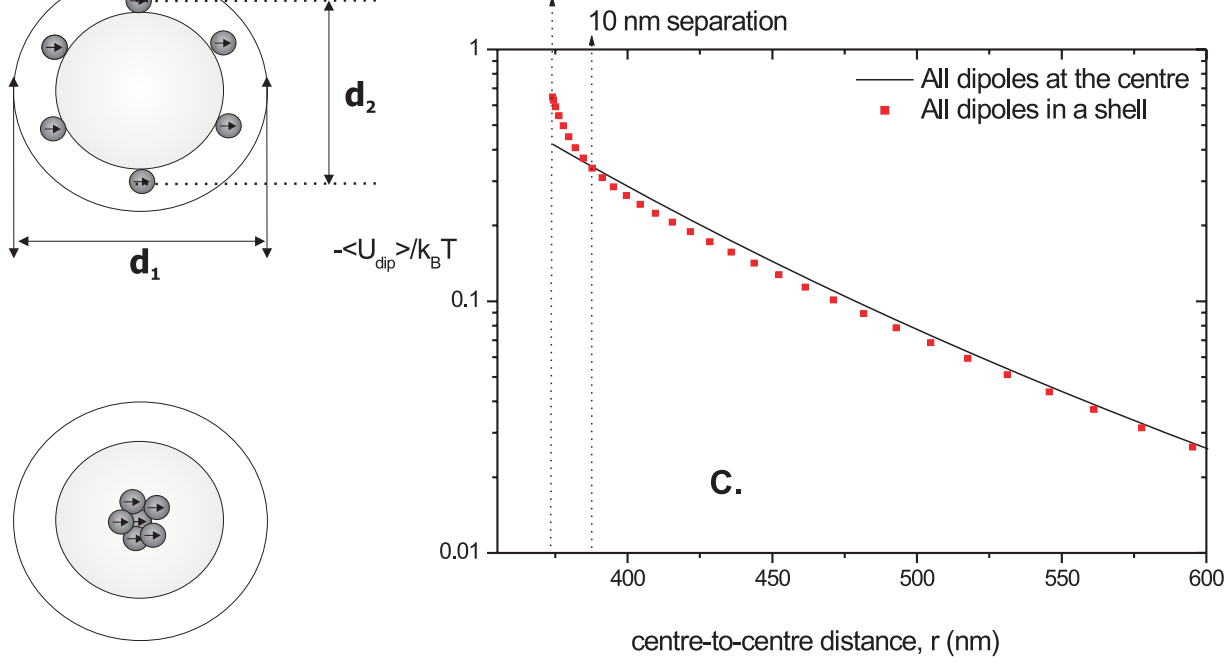

Figure 9. Left: schematic illustration to compare the attraction between composite colloidal particles of diameter $d_{1}$ with magnetic nanoparticles embedded (A) in a shell of diameter $d_{2}$ and (B) at the centre. Right: numerically calculated dipolar interaction potentials $\left\langle U_{\text {dip }}\right\rangle / k_{\mathrm{B}} T$ as a function of centre-to-centre distance between composite spheres as sketched in (A) and (B). The total diameter was chosen as $d_{1}=375 \mathrm{~nm}$ and the diameter of the shell of magnetic nanoparticles with 342 evenly distributed parallel magnetic dipoles adding up to a microparticle magnetic moment of $4 \times 10^{-17} \mathrm{~A} \mathrm{~m}^{2}$ was chosen as $d_{2}=355 \mathrm{~nm}$ and $d_{2}=0$, respectively. At a particle separation of $>10 \mathrm{~nm}$, the interaction potential for the silica particles with a shell of magnetic nanoparticles closely approximates the interaction potential for point dipoles.

calculated by integration across all possible orientations of both microparticles. The result is shown in figure 9(C). A control of the calculation is that, for $d_{2}=0$ (all nanoparticles at the centre, as in figure 9(B)), the numerical calculation agrees with (8) for point dipoles. A control of the sampling of configurations during numerical integration over different orientations is that the unweighted average interaction was found to be zero within digital accuracy. For a separation of the magnetic shells of $r=10 \mathrm{~nm}$, the result for $d_{2}=355 \mathrm{~nm}$ is almost indistinguishable from the calculation for $d_{2}=0$. Since the thickness of the outer silica layer of the studied particles already exceeds $10 \mathrm{~nm}$, we can conclude that it is a good assumption to treat the microparticle dipoles as point dipoles.

\subsection{Influence of dipolar interactions on rotational diffusion}

A clear result of our experiments is that the characteristic rotational frequency depends on the dipolar interactions between the particles (figure 4). When the permanent dipole moment of the particles is increased by magnetic treatment, the dipole-dipole interactions are enhanced and particle rotation slows down. However, two remarkable observations deserve discussion: the rotational retardation after dipole moment enhancement is only a weak function of the volume fraction above $\phi=0.01$, and salt addition does not decelerate rotation but accelerates it.

Before increasing the dipole moment of the microparticles (see figure 10, curve a), rotational diffusion is already somewhat slower than for hard spheres (see figure 10, curve b). If the increased dipole moments would only lead to further attraction between the particles, the rotational diffusion is expected to decrease more and more rapidly as a function of the volume fraction (curve $\mathrm{c}$ in figure 10). What is observed, however, is a fast initial retardation 


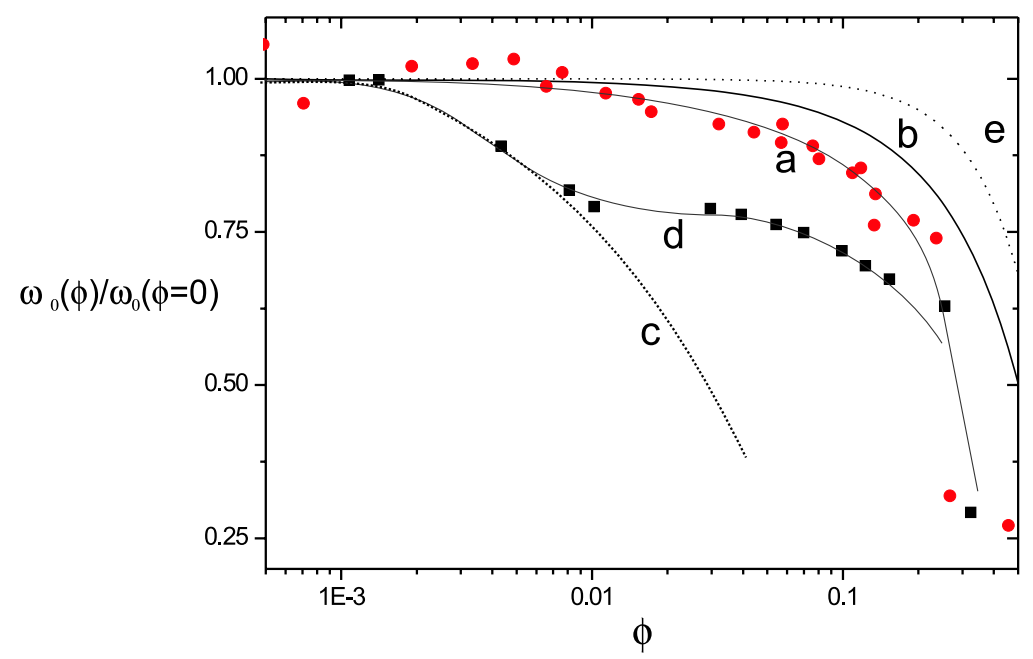

Figure 10. Comparison of experimental results of magnetic silica particles in sample A, before (a) and after (d) increasing the dipole moment, with calculated values for hard spheres (b, equation (2)) and charged spheres (e, equation (3)). Curve $\mathrm{c}$ is a hypothetical curve for the expected $\omega_{0}(\phi)$-profile due to dipole-dipole interactions only.

below a volume fraction of $\phi=0.01$, above which the rotation rate is no longer a strong function of volume fraction (curve $\mathrm{d}$ in figure 10). This result cannot be accounted for only on the basis of increased hydrodynamic interaction due to attraction-induced shortening of interparticle distances. One tempting explanation would be that, above $\phi=0.01$, the decrease in the time-averaged distance between neighbouring particles at increasing volume fraction is counteracted by electrostatic repulsion. This would set a minimum distance between interacting particles and thus limit retardation of rotation due to dipolar attraction. However, at a volume fraction of $\phi=0.01$, the average centre-to-centre interparticle distance is still of the order of $0.01^{-1 / 3} \sim 5$ particle diameters, whereas the Debye length in our systems without added salt is only $\sim 3 \%$ of one particle diameter (see section 3.1).

The length scales of the interactions are illustrated in figure 11, calculated on the basis of equations (4), (6), (8) and (9), using a particle radius of $187 \mathrm{~nm}$ and the measured ion concentrations in our system before and after salt addition. Typical values of the charge per surface area of TPM-coated Stöber-silica spheres in ethanol [28] were used and a Hamaker constant of silica in ethanol was estimated from an approximate expression given in [29]. Figure 11 illustrates that it is difficult to ascribe the relatively abrupt change in rotational dynamics around $\phi=0.01$ to electrostatic repulsion, when the average interparticle distance is still far below values where the electrostatic double layers of neighbouring particles are overlapping. Moreover, if electrostatic repulsion were to favour rapid rotation, salt addition should diminish that effect and lead to slower rotation due to the much increased attraction between the particles. Our experiments contradict this expectation: the addition of salt not only fails to slow down the rotation of particles, but even slightly enhances it.

It is concluded that, at concentrations above $\phi=0.01$, an effect is present that accelerates particle rotation at increasing colloidal concentration and that counteracts the retardation that is due to increasing hydrodynamic and dipolar interaction. On a microscopic level, there is apparently a mechanism by which the rotation of one particle facilitates that of its neighbours as the particles come closer together. To understand this, we propose that not only dipole-dipole 


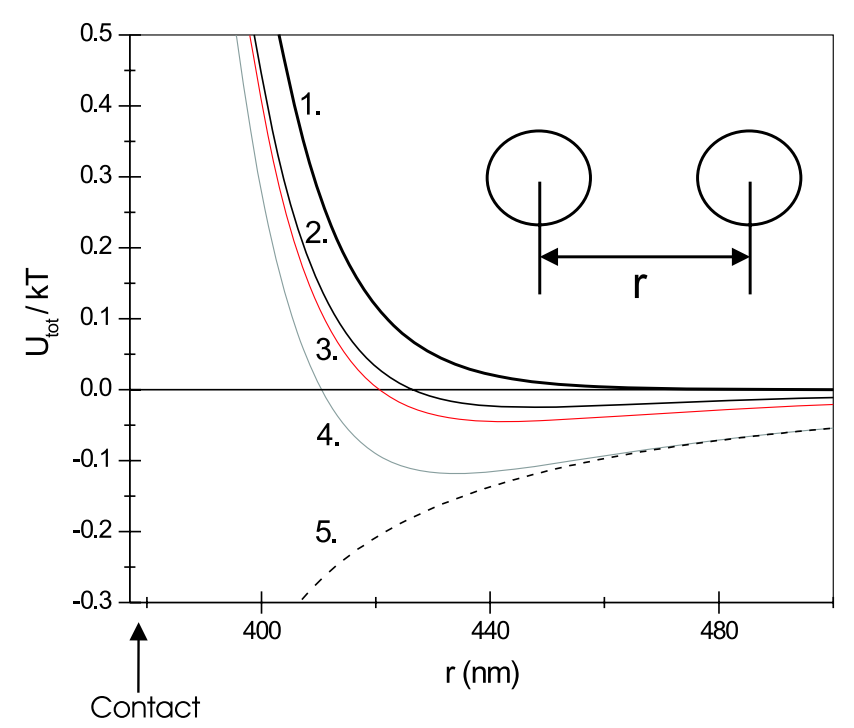

Figure 11. Calculated overall interaction potentials, $U / k_{\mathrm{B}} T$, as a function of the interparticle centre-to-centre distance, $r$, based on equations (4), (6), (8) and (9) for particles with $Z=1500$ [28], $\kappa^{-1}=12 \mathrm{~nm}, H \approx 3 \times 10^{-21} \mathrm{~J}$ and $R=187 \mathrm{~nm}: 1$, electrostatic repulsion; 2, electrostatic repulsion and van der Waals attractions; 3 and 4, electrostatic repulsion and van der Waals attractions in combination with dipole-dipole interaction for $\mu=2.5 \times 10^{-17} \mathrm{~A} \mathrm{~m}^{2}$ and $\mu=3.6 \times 10^{-17} \mathrm{~A} \mathrm{~m}^{2}$. Curve 5 is the same as in 4, but with a Debye length of $2 \mathrm{~nm}$, illustrating the effect of dispersing the particles in a $10 \mathrm{mM}$ monovalent salt solution.

interactions should be taken into account, but also that the interaction of the particle dipoles with the applied alternating magnetic field that probes the rotational motion of the particles.

Figure 12 sketches the time dependence of the orientation of the external magnetic field and of the dipolar particles. The applied field is up or down and its magnitude varies sinusoidally. The equipment measures the resulting magnetization of the sample, which depends on the orientations of the dipolar particles. Even when the strength of the applied field passes a maximum, particle alignment is only partial, since a weak magnetic field amplitude is used. When the field passes zero, the dipoles start to rotate towards the steady-state orientation distribution that corresponds to the instantaneous applied field. In table 2, values are given for the maximum dipolar interaction between the particles, $U_{\max }$, and the interaction energy, $\mu \mu_{0} H$, for the dipoles with the applied field. For the particles that are studied, the interaction with the field is on the order of $\mu \mu_{0} H=0.4-0.6 k_{\mathrm{B}} T$. Interaction with the field is only negligible when $\mu \mu_{0} H \ll k_{\mathrm{B}} T$, which is not the case. The alignment of the particle dipoles with the applied field can therefore not be regarded as purely diffusive.

The cooperative alignment of dipolar particles above a threshold concentration could be explained if doublets were formed. The interaction with the external field would be twice as strong as for single particles. This would explain why the increase in particle concentration (decrease in average distance between the particles) and addition of salt (decrease in distance of nearest approach) both lead to an acceleration of the particle rotation. However, as discussed in the previous section, the dipole-dipole interactions are not strong enough to form long-lived doublets or larger anisotropic structures. Nevertheless, the maximum dipole-dipole interaction for the particles in sample $\mathrm{A}$ is far from negligible: $\sim 0.6 k_{\mathrm{B}} T$ before and $\sim 1.2 k_{\mathrm{B}} T$ after magnetic treatment at $2 \mathrm{~T}$. Although not strong enough to form long-lived doublets, these particles could form short-lived doublets, as they come into contact with each other while 


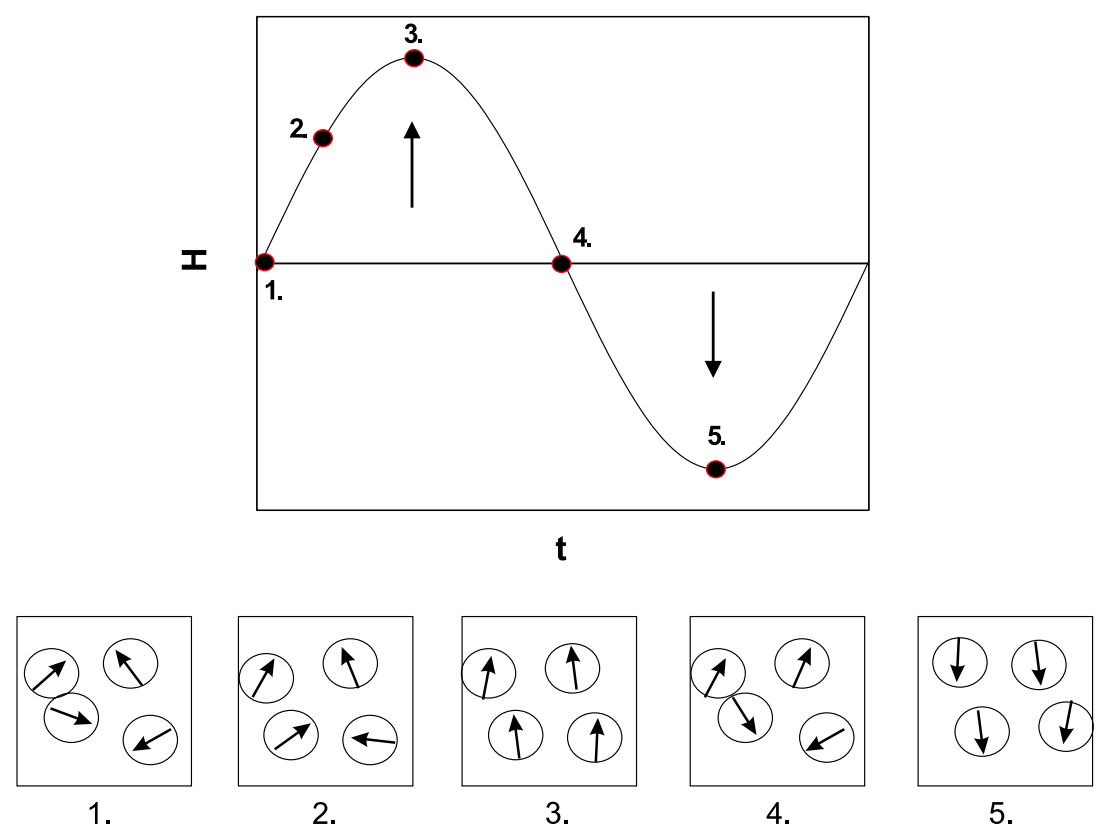

Figure 12. Illustration of the time dependence of the orientation of the external magnetic field and of the dipolar particles during complex magnetic susceptibility measurements. The equipment measures the resulting magnetization of the sample, which depends on the orientations of the dipolar particles. Even when the strength of the applied field passes a maximum, particle alignment is only partial, since a weak magnetic field amplitude is used.

diffusing around. The life-time of such a short-lived doublet can be roughly estimated based on the time that it takes for the particles to diffuse approximately one particle diameter apart after particle encounter. Particles with an attractive interaction potential of $\sim 1 k_{\mathrm{B}} T$ then have a doublet life-time on the order of $10 \mathrm{~ms}$. This is long enough to assist alignment with the field during half a period of the sinusoidally alternating field, leading to an enhanced particle rotation rate.

At the lowest concentrations, particle contact and the formation of short-lived doublets is much less frequent than at higher concentrations. The long-ranged dipole-dipole interactions can slow down the rotational diffusion and shorten the average distance between the particles, thereby enhancing the hydrodynamic interactions in the system.

Our explanation implies that particle rotation is no longer purely diffusive in our systems above $\phi=0.01$, but that it is assisted by the external field and by dipole-dipole interactions. Below $\phi=0.01$, the concentration is low enough for the rotation of particles to be purely diffusive. In future applications, when the objective is to measure the rotational diffusion rate at high concentrations of the magnetically labelled particles $(\phi>0.01)$, the magnetic interaction should remain weaker than in system A after magnetic treatment. This suggests that the headto-tail contact interaction energy should be kept well below $k_{\mathrm{B}} T$ to use the particles as probes of rotational diffusion at high concentrations. To realize this, lower amounts of or less strongly magnetic material should be used and the magnetic nanoparticles should be embedded far enough below the surface of the microparticles to avoid enhanced dipolar interaction (figure 9). Moreover, the magnitude of the alternating magnetic field could be decreased even further as long as the signal-to-noise ratio remains sufficiently high. 


\section{Conclusions}

We have demonstrated that frequency-dependent complex magnetic susceptibility measurements can be employed as a new experimental method to study rotational diffusion in colloidal systems, provided that the particles are labelled with thermally blocked magnetic dipoles and that the particle dipole moment remains small (head-to-tail contact interaction $\ll k_{\mathrm{B}} T$ ). This method has the advantage that it can be applied to colloidal particles of various shapes and in the size range of 5-500 $\mathrm{nm}$ in radius, without refractive index matching. Even strongly light scattering or absorbing materials can be studied. The technique allows direct measurements of the rotational diffusion coefficient and the width of its distribution over the full range of volume fractions without using host-tracer systems. Moreover, colloidal particles with attractive dipolar and/or van der Waals interactions can be studied, since this magnetic technique is not sensitive to optical scattering or absorption. This opens up possibilities to examine the rotational diffusion of a variety of complex colloidal systems such as anisotropic and/or dipolar particles as well as particles in various solvents or confined geometries.

Using this technique we have been able to perform an experimental study of the concentration dependence of the Brownian rotation for charged colloidal silica spheres with an intrinsic, permanent dipole moment. The results show that dipolar interactions between the particles lead to a faster initial decay of the rotational diffusion coefficient as a function of the volume fraction than for hard spheres or charged spheres. At volume fractions $\phi>0.01$, magnetic coupling between the dipole moments accelerated particle rotation in the alternating external field, and rotation was no longer purely diffusive.

We have also shown that a slightly thicker outer shell partially screens the magnetic interactions, which means that, by growing an outer non-magnetic layer, the particles eventually behave as charged spheres and the magnetic particles are only acting as a probe for the rotational diffusion measurements.

\section{Acknowledgment}

This work was financially supported by OCÉ Technologies, Venlo, The Netherlands.

\section{References}

[1] Kerker M 1969 Scattering of Light and other Electromagnetic Radiation (New York: Academic)

[2] Piazza R, Degiorgio V, Corti M and Stavans J 1990 Phys. Rev. B 424885

[3] Degiorgio V, Piazza R and Jones R B 1995 Phys. Rev. E 522707

[4] Piazza R and Degiorgio V 1991 Phys. Rev. Lett. 673868

[5] Lettinga M P, van Kats C M and Philipse A P 2000 Langmuir 166166

[6] Lettinga M P, van Zandvoort M A M J, van Kats C M and Philipse A P 2000 Langmuir 166156

[7] Koenderink G H, Lettinga M P and Philipse A P 2002 J. Chem. Phys. 1177751

[8] Lettinga M P, Koenderink G H, Kuipers B W M, Bessels E and Philipse A P 2004 J. Chem. Phys. 1204517

[9] Sashchiuk A, Amirav L, Bashouti M, Krueger M, Sivan U and Lifshitz E 2004 Nano Lett. 4159

[10] Zhang H and Nägele G 2002 J. Chem. Phys. 1175908

[11] Hagen M H J, Frenkel D and Lowe C P 1999 Physica A 272376

[12] Watzlawek M and Nägele G 1997 Prog. Colloid Polym. Sci. 168168

[13] Claesson E M and Philipse A 2005 Langmuir 219412

[14] Claesson E M, Erné B H, Bakelaar E and Kuipers B M W 2007 J. Phys.: Condens. Matter 19036105

[15] Cichocki B, Ekiel-Jezewska M L and Wajnryb E 1999 J. Chem. Phys. 1113265

[16] Atkins P W 1998 Physical Chemistry 6th edn (Oxford: Oxford University Press)

[17] Lide D R (ed) 2000 Handbook of Chemistry and Physics (Boca Raton, FL: CRC Press)

[18] Fannin P 1998 Adv. Chem. Phys. 104181 
[19] Tourinho F A, Franck R and Massart R 1990 J. Mater. Sci. 253249

[20] Stöber W, Fink A and Bohn E 1968 J. Colloid Interface Sci. 2662

[21] Kuipers B W M, Bakelaar I A, Klokkenburg M and Erné B H 2007 in preparation

[22] Chen D-X, Brug J A and Goldfarb R B 1991 IEEE Trans. Magn. 273601

[23] Erné B H, van der Pol E, Vroege G J, Visser T and Wensink H H 2005 Langmuir 211802

[24] Philipse A and Maas D 2002 Langmuir 189977

[25] Klokkenburg M, Dullens R P A, Kegel W K, Erné B H and Philipse A P 2006 Phys. Rev. Lett. 960372031

[26] Klokkenburg M, Erné B H, Meeldijk J D, Wiedenmann A, Petukhov A V, Dullens R P and Philipse A P 2006 Phys. Rev. Lett. 97185702

[27] Erné B H, Butter K, Kuipers B W M and Vroege G J 2003 Langmuir 198218

[28] Philipse A and Vrij A 1989 J. Colloid Interface Sci. 128121

[29] Bergna H E (ed) 1994 The Colloid Chemistry of Silica (Advances in Chemistry Series vol 234) (Washington, DC: American Chemical Society) 